\title{
Chromatic confocal setup for displacement measurement using a supercontinuum light source
}

\author{
U. Minoni*, G. Manili, S. Bettoni, E. Varrenti, D. Modotto, C. De Angelis \\ Dipartimento di Ingegneria dell'Informazione, Università degli Studi di Brescia, Via Branze 38, Brescia 25123, Italy
}

\section{A R T I C L E I N F O}

\section{Article history:}

Received 31 August 2012

Received in revised form

10 October 2012

Accepted 1 November 2012

Keywords:

Chromatic confocal setup

Displacement measurement

Supercontinuum

\begin{abstract}
A B S T R A C T
Chromatic confocal microscopy is a technique to measure distances by analyzing the spectrum of the light reflected by a sample. The key element of the confocal setup is a dispersive lens, which focuses different wavelengths at different distances from the lens. In this paper, a novel setup realized with a supercontinuum light source and a spatial filter composed by reflective elements is described. The supercontinuum source is implemented by injecting high power pulses from a microchip laser into a Ge-doped microstructured optical fiber. The usage of metallic parabolic mirrors, for the focusing and collimation required in the spatial filter, lets the dispersive lens be the only dispersive element of the confocal setup and improves the efficiency of the spatial filter itself. A silicon-based spectrometer is used for the acquisition of the spectra, which are normalized and Gaussian-fitted before extracting the displacement information. A complete calibration is performed, and the set of wavelengths from $500 \mathrm{~nm}$ to $900 \mathrm{~nm}$ can be mapped into a $280 \mu \mathrm{m}$ measuring range. The obtained relative accuracy of $0.36 \%$ shows an enhancement of almost one order of magnitude when compared to other supercontinuum-based confocal systems.
\end{abstract}

(c) 2012 Elsevier Ltd. All rights reserved.

\section{Introduction}

Confocal microscopy is a well-known technique characterized by the capability to localize the position of a point on the object surface along the system optical axis. The distance of the point is determined by measuring the maximum detected intensity at the photo-detector while moving the object or the measuring head. The chromatic variant of the confocal technique [1] enables us to avoid the axial translation, making it possible to build a faster and more reliable system. With this configuration the position is determined by the analysis of the spectrum of the light exiting the confocal setup: the distance of the surface point is mapped to a peak position on the optical spectrum. Recently, results based on the use of a broadband lamp as the light source have been presented [2-4]: the measurement range varies from few tens of micrometers [2] up to few millimeters [3]. The best relative accuracy over a range of $400 \mu \mathrm{m}$ was obtained by using a Gaussian fitting of the spectrum data [4].

The introduction of a supercontinuum (SC) [5] light source was proposed a few years ago with the objective to obtain both a wider measuring range and a better lateral resolution of the measurement [6,7]. In fact, the SC is characterized by an extended wavelength range and when it is transported by the fundamental mode of a microstrucured optical fiber the SC can be assumed

\footnotetext{
* Corresponding author. Tel.: +39030 37151; fax: + 39030380014 .

E-mail address: umberto.minoni@ing.unibs.it (U. Minoni).
}

perfectly spatially coherent. The published works, however, have shown measuring results which in some respects are worse than the ones obtained with a lamp source. Taking as an example the relative accuracy of the displacement measurement, its value ranges from about $10 \%$ [6] to 7.5\% [8] and becomes no better than a few percents [7], whilst the same value is steadily lower than $1 \%$ when the source used is a lamp [1,2].

The full exploitation of the SC potential in a chromatic confocal system implies the use of the very wide wavelength range provided by the light source. On the other hand, over a wide wavelength range the residual dispersion of the optical components can significantly contribute to worsening the confocal condition and therefore deteriorate the quality of the measurement.

In this paper we present a displacement measuring system based on a chromatic confocal setup where the light source is a SC generator built around a microstructured optical fiber. To confine the required chromatic aberration to just one optical element, that is the dispersive lens, the spatial filtering of the light was performed with the use of metallic reflective optics. A highly nonlinear microstructured optical fiber (MOF) has been chosen in order to provide a suitable spectrum smoothly covering the wavelength range from $450 \mathrm{~nm}$ up to $900 \mathrm{~nm}$.

\section{Supercontinuum generation}

SC generation can take place whenever high power optical pulses propagate inside a nonlinear medium, such as an optical 
fiber. This phenomenon, due to the joint action of the nonlinearities of the medium and its dispersive properties, was first observed in bulk media in 1970s [9], but it became widespread after the introduction of MOFs [10]. These waveguides are silica fibers characterized by a regular lattice of air-holes running along the axis of the fiber itself; the introduction of defects in the air-hole array makes possible the guidance of light. MOFs enable to choose with relatively high freedom the dispersive properties of the guiding cores by changing both the design of this structure [11] and the doping [12] of the cores. For the SC source employed in the experiment, we used one out of the seven cores of a MOF, whose characteristics have been reported by Modotto et al. [13]. The core was doped with germanium, with a concentration which led to a refractive index increase $\Delta n=0.0125$, and was one of the two in line with the central core (see ref. 13 and figures therein).

The SC was generated by injecting 600-ps-long pulses from a microchip laser in a $3 \mathrm{~m}$ piece of MOF by means of the micro-lens $\mathrm{L} 1$, having a focal length of $3.3 \mathrm{~mm}$. The power coming from the laser could be changed by rotating the neutral density filter ND, and the mirrors M1 and M2 were used to optimize the angle of the light entering the micro-lens (see Fig. 1). The polarization of the beam was controller by the $\lambda / 2$ plate and the output was collected and collimated by a $20 \times$ achromatic microscope objective indicated as L2 in Fig. 1.

\section{Confocal setup}

The displacement measurement, based on the chromatic confocal technique, was implemented by realizing the setup represented in Fig. 2. The SC light, generated as explained in Section 2, is used to illuminate a confocal setup which is followed by a spectrometer, used to acquire the spectra. The beam provided by the light source, after passing through the beam splitter BS, was focused onto the target mirror $\mathrm{M}$ by means of the dispersive lens DL. For the experiments we used a $\mathrm{CaF}_{2}$ lens (Thorlabs LB5766) having a focal length of $15 \mathrm{~mm}$ at the nominal wavelength of $588 \mathrm{~nm}$, whose focal length shift, in the spectral range from $488 \mathrm{~nm}$ to $1064 \mathrm{~nm}$, was $280 \mu \mathrm{m}$. The light reflected by the sample passed through the same lens and then propagated into the beam splitter, which sent part of the returning light towards a spatial filter. The latter was composed by the two metallic parabolic mirrors PM1 and PM2 and the pinhole PH. The two parabolic mirrors were placed one in front of the other, and aligned so that the two focuses were coincident and overlapped with the pinhole aperture, as shown in Fig. 2.

The choice to build the spatial filter by means of reflective optics was due to the necessity to guarantee an achromatic behavior of the filter itself. For the experiments we used two parabolic mirrors having an effective focal length of $50.80 \mathrm{~mm}$ (Edmund Optics 47097) and a pinhole having a diameter of $10 \mu \mathrm{m}$. The decision of using metallic parabolic mirrors came from preliminary experiments. In fact

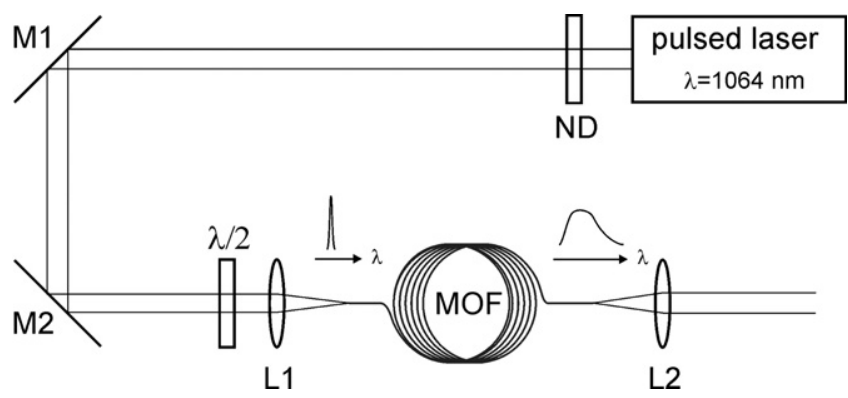

Fig. 1. Setup of the SC source. The light coming from the microchip laser propagates into a neutral density filter ND, and reflects on the mirrors M1 and M2. The $\lambda / 2$ plate is used to control the polarization of the beam, and the lens L1 focuses the laser light into the MOF. The objective L2 is used for collimation.

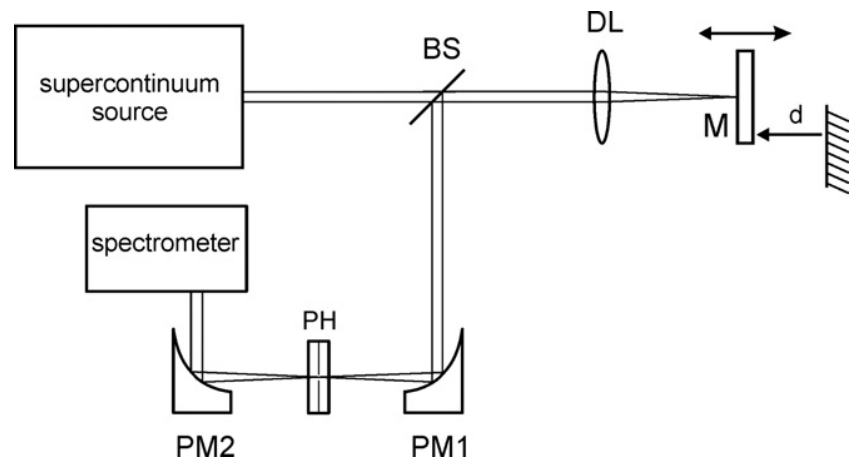

Fig. 2. Setup of the confocal system. The light coming from the supercontinuum source is focused on the mirror M by the dispersive lens DL. A beam splitter BS sends part of the reflected light to the spatial filter, composed by the metallic parabolic mirrors PM1 and PM2 and the pinhole PH. The filtered light is collected by a spectrometer.

obtaining a wavelength-independent spatial filter including only refractive optics would increase the level of complexity and the number of components. The light exiting the spatial filter was collected by a spectrometer, used to measure the filtered output of the system. With the exception of the lens DL, all the optical components of the setup were achromatic. Therefore, since only the focal length of the lens DL was dependent on the optical wavelength $\lambda$, the confocal condition could be easily tuned for the different wavelengths by simply adjusting the axial position of the target mirror $\mathrm{M}$. The position of the mirror $\mathrm{M}$ is indicated as $\mathrm{d}$ in Fig. 2, and was measured with respect to an arbitrary reference. In other words, the peak intensity of the light entering into the spectrometer was centered at a specific wavelength depending on the distance of target mirror $M$ from lens DL. After a suitable calibration it was therefore possible to measure the target mirror displacements by tracing the peak wavelength of the optical spectrum of the light captured by the spectrometer.

For the experiments we used the USB2000 spectrometer from Ocean Optics capable of measuring over a wavelength range from $350 \mathrm{~nm}$ to $1025 \mathrm{~nm}$.

\section{Experimental results}

\subsection{Source characterization}

The first measurements focused on the characterization of the optical beam provided by the SC generator. The average diameter of the beam was of about $2 \mathrm{~mm}$. The optical spectrum as measured by the spectrometer is depicted in Fig. 3; the spectrometer readout has been normalized to its maximum value in order to better represent the optical spectrum. It is important to note that the spectrometer used for the experiments is equipped with a CCD linear optical sensor (Sony ILX511) characterized by a peak of the spectral sensitivity around $450 \mathrm{~nm}$ and a spectral sensitivity decreasing with the longer wavelengths, at $900 \mathrm{~nm}$ the relative sensitivity is $15 \%$ and at $1 \mu \mathrm{m}$ it is only a few percent. The vertical scale of the drawing of Fig. 3 has not been corrected to compensate for the different sensitivity at different wavelengths, therefore the vertical units cannot be expressed in SI system units.

\subsection{Displacement determination algorithm}

The target mirror position measurement is based on the acquisition of the optical spectrum coming from the spatial filter and the application of a number of processing steps. The basic 


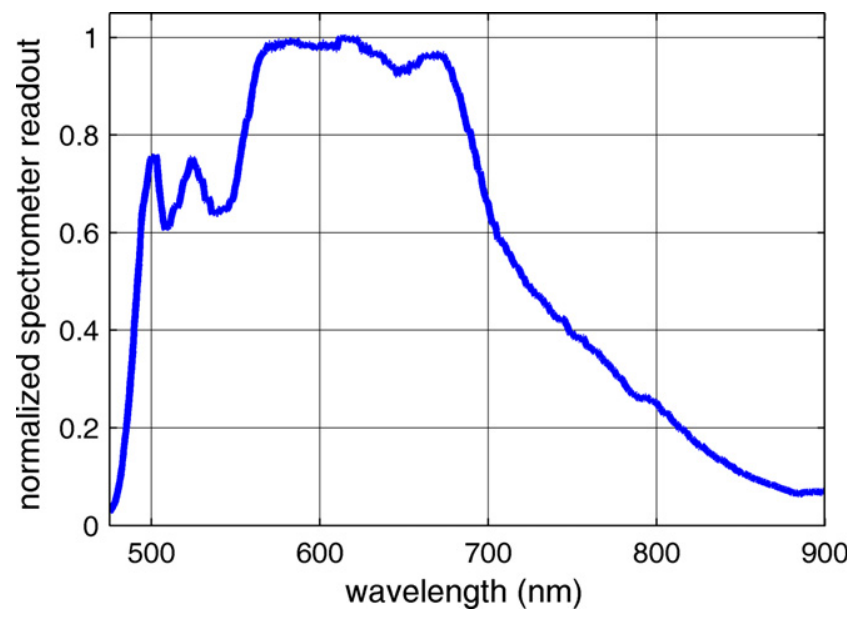

Fig. 3. Spectrum of the SC generated by the setup of Fig. 1 as measured at the output of the collimating lens L2 of Fig. 1.

idea is to identify the optical wavelength at which the confocal system is actually tuned. The task is very easy when the spectrum of the light source is flat, since the spectrum at the output of the optical setup mimics the transfer function of the spatial filter, which presents a maximum peak centered on the wavelength which is most efficiently focused onto the target mirror. By changing the relative position of the mirror with respect to the dispersive lens, the central peak of the spectrum moves at different wavelengths. As evident from Fig. 3, the actual spectrum of the SC source was not flat, and therefore it was necessary to perform some processing before being able to determine the wavelength at which the confocal system was tuned. The first step was the acquisition of a reference spectrum, obtained by removing from the optical setup the dispersive element (the lens DL of Fig. 2). This reference spectrum slightly differs from the one of the SC source as it takes into account for the effect of all the optical components in the setup (excluded the diffractive lens). The comparison of spectra acquired when the lens is inserted with the reference spectrum makes it feasible to understand the effect of the dispersive element on the confocal condition.

In Fig. 4 the reference spectrum is represented as a blue line. In the same figure a few spectra acquired at different target positions are superimposed on the reference spectrum. The distance $d$ tagging the different spectra corresponds to the mirror displacement measured with respect to a fixed reference position (see Fig. 2). From Fig. 4 it is possible to note that the optical signal significantly increases with distance $d$, that is, with the confocal condition moving towards the shorter wavelengths where the intensity of the reference signal is stronger. To better estimate the central wavelength of each spectrum we applied to each point of the spectra the following transformation:

$I_{\text {rel }}(\lambda)=\frac{I_{\text {spect }}(\lambda)-I_{\text {dark }}(\lambda)}{I_{\text {ref }}(\lambda)-I_{\text {dark }}(\lambda)}$

$I_{\text {spect }}(\lambda)$ is the light power density as measured by the spectrometer, $I_{\text {dark }}(\lambda)$ is the dark power density (noise), $I_{\text {ref }}(\lambda)$ is the power density of the reference spectrum, and $I_{\text {rel }}(\lambda)$ is the computed relative power density. Fig. 5 shows the results of this first elaboration applied to the spectra of Fig. 4: it is evident the improvement of the signal quality from the point of view of the peak wavelength extraction.

Finally, the spectra of Fig. 5 were normalized in amplitude and subsequently fitted with a Gaussian curve [4], obtaining the curves reported in Fig. 6. The positions of the target mirror can be inferred from the wavelengths at which the Gaussian fitting curves are centered.

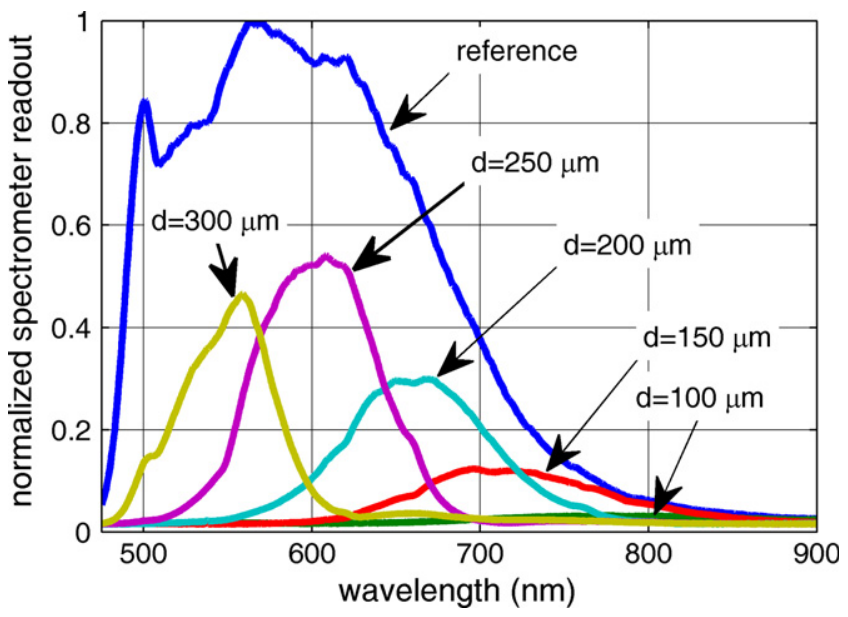

Fig. 4. A number of spectra as acquired after the spatial filtering: reference is the spectrum obtained without the dispersive lens, the distance associated to the other spectra indicates the target relative displacement.

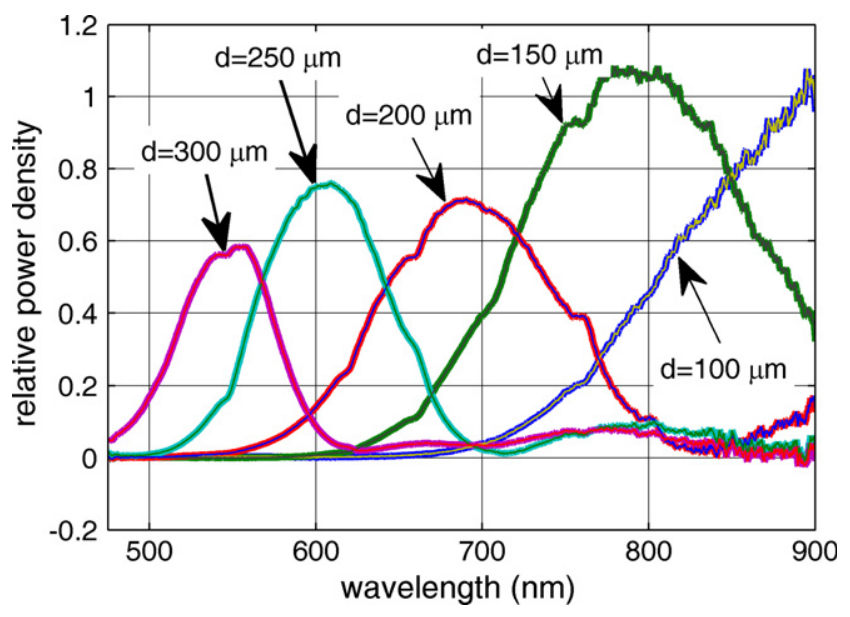

Fig. 5. Power densities normalized to the reference spectrum.

The proposed signal elaboration algorithm resulted robust to the optical source spectral non-uniformity and fluctuations, provided a very good sensitivity also when the signal become weak, and let us extend the measuring range towards the infrared region up to the limit of the spectrometer capability.

\subsection{System calibration}

The calibration of the system was obtained by moving the target mirror to a number of reference distances and registering and elaborating the corresponding spectrometer signal. With reference to Fig. 2, the mirror $M$ was mounted on a linear slide whose position was controlled by a micrometric screw. The displacement d was measured from a conventional "zero" position located far from the lens. Increasing distances corresponded to movements towards the lens DL. Fig. 7 reports the calibration points as empty diamonds; superimposed is the second order polynomial calibration fitting curve. The measuring range is of about $0.28 \mathrm{~mm}$. Both the lower and the upper bounds of the measuring range are restricted by the concurrent contribution of the spectral characteristic of the optical source and by the spectral range and sensitivity of the spectrometer. The standard deviation of the experimental data from the calibration curve, computed over the entire measuring range, is of $6 \mu \mathrm{m}$.

In order to assess the capability of the instrument to provide reliable measures we estimated the repeatability of the system: 


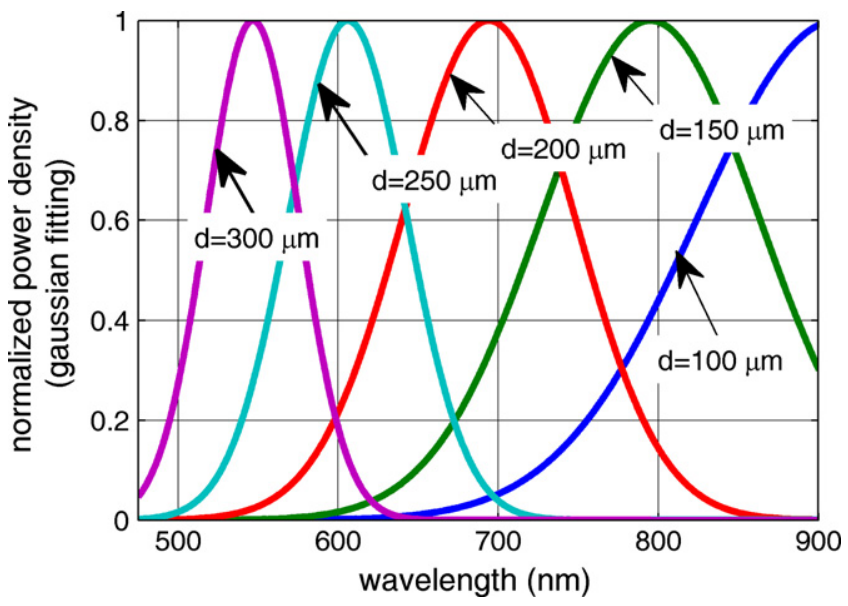

Fig. 6. Gaussian interpolation of the spectra of Fig. 5.

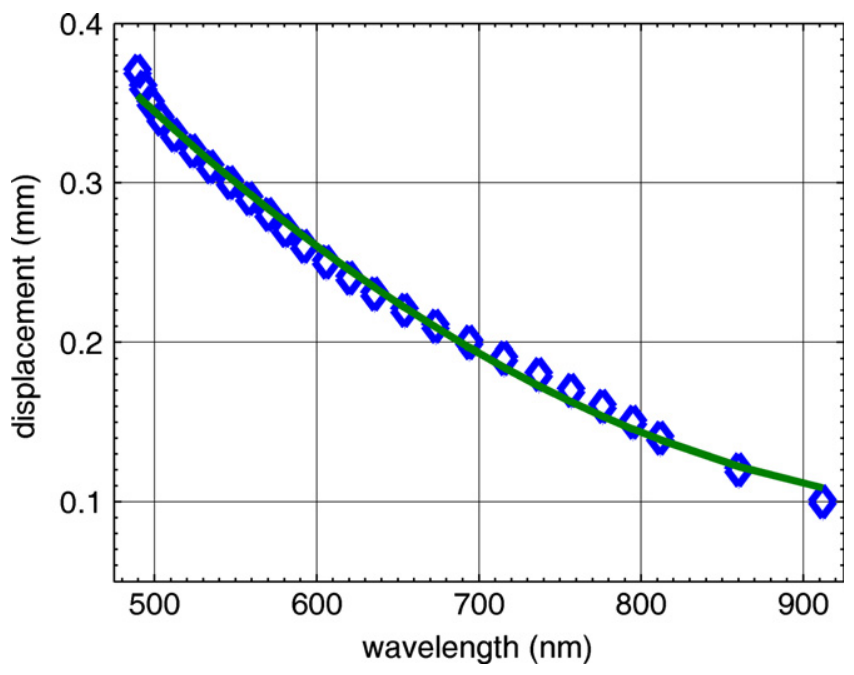

Fig. 7. Calibration of the confocal displacement meter. The continuous line represents the interpolation of the experimental data with a parabolic curve.

an arbitrary point in the middle of the measuring range has been chosen and the target mirror was repetitively positioned to that point starting from a different position. As an example of the results, in Fig. 8 the deviations of the target position measurements from the mean value are reported for 20 successive repositionings: the repeatability of the measurement is smaller than $1 \mu \mathrm{m}$. This value is very good since it is of the same order of magnitude of the uncertainty of the micropositioner used to move the target mirror. In other words, we can state with a good confidence that the repeatability of the measuring technique is better than that of the setup used to estimate the repeatability itself.

Assuming the repeatability as a representation of the main source of uncertainty, the relative accuracy of the prototype can be estimated as the ratio of the repeatability itself over the measurement range, resulting equal to $0.36 \%$.

\section{Conclusion}

A chromatic confocal setup for displacement measurement is proposed based on the use of a SC light source and an achromatic

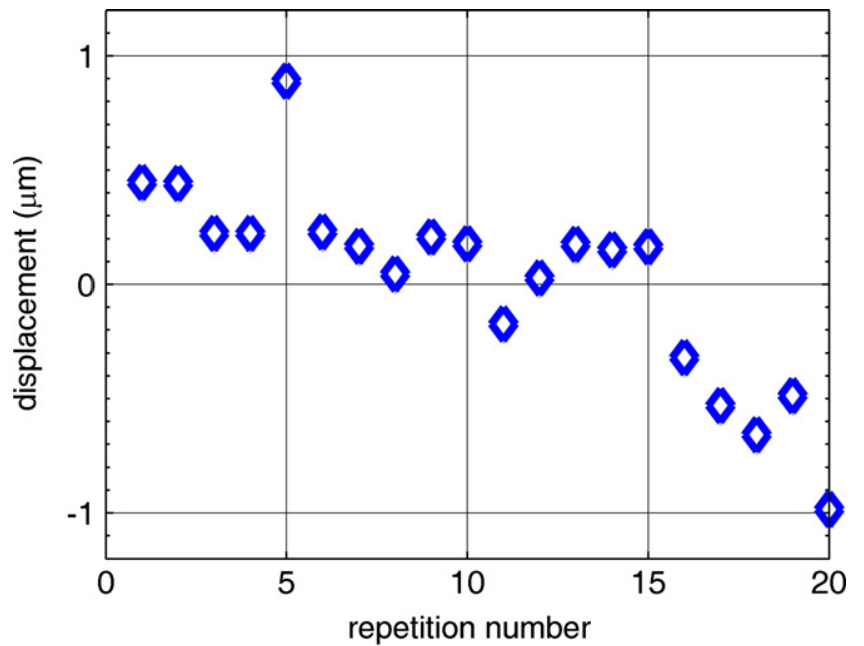

Fig. 8. Example of measurements repeated by moving the target mirror back and forth around a predefined position.

spatial filter. The setup in combination with the mentioned data processing algorithm is capable of making measurements over an optical wavelength range extending from $500 \mathrm{~nm}$ up to $900 \mathrm{~nm}$. The experiments showed a relative measurement accuracy better than $0.36 \%$ over a displacement range of $280 \mu \mathrm{m}$. The prototype showed results which are better than those obtained with previous SC-based systems, and comparable with lamp-based systems. Further work will be directed to extend the optical wavelength range into the near infrared region in order to get a wider measuring range.

\section{References}

[1] Molesini G, Pedrini G, Poggi P, Quercioli F. Focus-wavelength encoded optical profilometer. Optics Communications 1984;49:229-33.

[2] Chun BS, Kim K, Gweon D. Three-dimensional surface profile measurement using a beam scanning chromatic confocal microscope. Review of Scientific Instruments 2009;80 07376-1.

[3] Niu CH, Deng WY, Mao XH, Gao X, Han C. Theoretical and experimental study on chromatic confocal position sensor, Proc. of SPIE, 7853 (2010) 78531B1-8.

[4] Luo D, Kuang C, Liu X. Fiber-based chromatic confocal microscope with Gaussian fitting method. Optics \& Laser Technology 2012;44:788-93.

[5] Dudley JM, Genty G, Coen S. Supercontinuum generation in photonic crystal fiber. Reviews of Modern Physics 2006;78:1135-84.

[6] Shi K, Li P, Yin S, Liu Z. Chromatic confocal microscopy using supercontinuum light. Optics Express 2004;12:2096-101.

[7] Garzon J, Meneses J, Tribillon G, Gharbi T, Plata A. Chromatic confocal microscopy by means of continuum light generated through a standard single-mode fibre. Journal of Optics A: Pure and Applied Optics 2004;6: 544-8.

[8] Shi K, Nam SH, Li P, Yin S, Liu Z. Wavelength division multiplexed confocal microscopy using supercontinuum. Optics Communications 2006;263: 156-62.

[9] Alfano RR, Shapiro SL. Emission in the region 4000 to $7000 \AA$ via four-photon coupling in glass. Physical Review Letters 1970;24:584-7.

[10] Eggleton BJ, Kerbage C, Westbrook PS, Windeler RS, Hale A. Microstructured optical fiber devices. Optics Express 2001;9:698-713.

[11] Labruyère A, Martin A, Leproux P, Couderc V, Tonello A, Traynor N. Controlling intermodal four-wave mixing from the design of microstructured optical fibers. Optics Express 2008;16:21997-2002.

[12] Labruyère A, Leproux P, Couderc V, Tombelaine V, Kobelke J, Schuster K, et al. Structured-core $\mathrm{GeO}_{2}$-doped photonic-crystal fibers for parametric and supercontinuum generation. IEEE Photonics Technology Letters 2010;22: 1259-61.

[13] Modotto D, Manili G, Minoni U, Wabnitz S, De Angelis C, Town G, et al. Gedoped microstructured multicore fiber for customizable supercontinuum generation. IEEE Photonics Journal 2011;3:1149-56. 